\title{
La limite de puissance de l'Union Européenne dans les relations internationales : les divergences et les convergences de l'UE avec les États-Unis, en particulier depuis l'arrivée au pouvoir de Donald Trump, sur l'Accord du nucléaire iranien de 2015 : le Joint Comprehensive Plan of Action (JCPOA) Kamal Bayramzadeh ${ }^{1}$
}

\section{Résumé}

Cet article se donne pour objectif de montrer le rôle de l'Union Européenne (UE) dans la résolution des crises internationales, en l'occurrence la crise nucléaire iranienne. L'UE est dotée d'une «politique étrangère commune » depuis 1993 et, en raison de sa capacité d'influence politique et économique dans certains dossiers internationaux, elle peut être qualifiée d'acteur international. Nous voulons examiner les différentes dimensions de la puissance européenne afin de mettre en évidence la limite de sa puissance normative en nous focalisant sur la crise nucléaire iranienne et les divergences de l'UE avec les États-Unis depuis l'arrivée au pouvoir de Donald Trump. Après avoir expliqué les concepts analytiques de puissance douce, de puissance normative et d'acteur, nous allons d'abord mettre en exergue les caractéristiques de la puissance de l'UE. Cette étude va ensuite analyser les différents aspects de la politique de l'UE à l'égard de l'Iran et en particulier son projet nucléaire de 1992 à 2020. Nous mettrons finalement en évidence les impacts de l'arrivée au pouvoir de Donald Trump sur les relations des États-Unis avec l'UE. Il s'agit de montrer comment la nouvelle approche politique et économique des États-Unis (néomercantilisme) sous la présidence de D. Trump a changé l'orientation de la politique étrangère des États-Unis en particulier à l'égard de l'UE et de l'accord nucléaire de 2015 du P5+1 avec 1'Iran. En 2018, les États-Unis se sont retirés de l'accord et ont imposé leur loi extraterritoriale aux entreprises étrangères en interdisant de commercer avec l'Iran. En dépit de sa protestation, l'UE n'a pas pu empêcher que cette loi soit imposée aux entreprises européennes, ce qui a illustré la limite de sa puissance.

Mots-clés : Iran ; Union Européenne ; États-Unis ; Crise nucléaire iranienne ; Donald Trump ; Puissance normative ; Néomercantilisme ; Dialogue critique ; Loi extraterritoriale

\footnotetext{
${ }^{1}$ Kamal Bayramzadeh est enseignant en Relations internationales à l'Université Sorbonne Paris Nord (Paris 13) et chercheur invité au Département de Science politique de l'Université de Liège (ULiège). Il est membre associé de l'Institut de Droit Public, Sciences Politiques et Sociales de Paris 13 (IDPS), membre du Center for International Relations Studies (CEFIR) (ULiège) et membre associé de la Chaire Raoul-Dandurand en études stratégiques à l'Université du Québec à Montréal (UQAM), Observatoire sur le Moyen-Orient et l'Afrique du Nord.
} 


\section{Introduction}

L'accession à la présidence américaine de Donald Trump a contribué à la dégradation des relations entre l'Union européenne (UE) et les États-Unis en raison d'une nouvelle approche en matière de politique étrangère, axée plus particulièrement sur l'unilatéralisme, et d'une nouvelle politique économique liée au néomercantilisme ${ }^{1}$. Depuis son installation, le nouveau président n'a pas manqué de qualifier la Chine, la Russie et l'UE d'ennemies. L'un des problèmes majeurs qui a révélé les divergences entre l'UE et les États-Unis est le retrait de ces derniers de l'accord multilatéral du Plan global d'action conjoint portant sur le projet nucléaire iranien. Non seulement la première puissance mondiale s'est retirée en 2018 de l'Accord, mais au demeurant, elle a instauré une loi extraterritoriale interdisant aux autres pays de commercer avec l'Iran, et a contraint les entreprises européennes de se retirer d'Iran. En dépit de l'importance de cet Accord pour elle, l'UE a montré la limite de sa puissance et de sa souveraineté économique vis-à-vis des États-Unis, alors qu'elle avait joué un rôle essentiel en tant qu'acteur de médiation et de négociation dans sa conclusion en 2015.

L'UE avait noué des relations avec l'Iran dans le cadre du dialogue critique (1992) et dans le dialogue global (1998) en insistant sur plusieurs conditions ; notamment la nonprolifération des armes de destruction massive. Durant des années, l'UE a choisi de dialoguer et de coopérer avec l'Iran en utilisant sa puissance normative (normative power), alors que les États-Unis ont opté pour une politique de sanction et de pression économique afin de limiter la puissance iranienne au Moyen-Orient. Mais depuis 2010, l'UE a changé sa stratégie et mis en œuvre la politique de l'arme économique ${ }^{2}$ afin d'amener l'Iran à la table de négociations pour y traiter de l'accélération de son projet nucléaire. Dans ce contexte international, l'avènement du conflit syrien et l'implication de l'Iran pour défendre le régime de Bachar el-Assad ont contribué au changement des rapports de force sur le plan régional en faveur de Téhéran; ce nouvel équilibrage n'ayant pas manqué d'inquiéter d'autres puissances du Moyen-Orient dont l'Arabie Saoudite et Israël. À partir de 2013, quatre facteurs ont marqué la poursuite des négociations multilatérales relatives au nucléaire iranien. Premièrement, l'élection d'un nouveau président en Iran (Hassan Rohani) favorable à la négociation avec les pays occidentaux. Deuxièmement, les effets des sanctions économiques contre l'Iran. Troisièmement, la montée en puissance de l'Iran au Moyen-Orient. Quatrièmement, le changement de l'approche américaine relative au nucléaire iranien qui sous la présidence de Barack Obama épousa fortement celle de $1^{\prime} U^{3}, c^{\prime}$ 'est-à-dire reconnaître à l'Iran le droit d'accéder au nucléaire civil. Cette approche engendra des négociations directes entre les ÉtatsUnis et l'Iran et transforma la crise nucléaire iranienne en objectif des négociations multilatérales entre le groupe $\mathrm{P} 5+1$ et 1 'Iran. Ces efforts diplomatiques aboutirent à l'Accord de 2015 qualifié d'historique par les acteurs concernés en dépit de l'opposition d'Israël et de l'Arabie Saoudite.

Cet article se donne pour objectif d'analyser le rôle de l'UE dans la résolution des crises internationales, en l'occurrence celle du nucléaire iranien. L'UE est dotée d'une " politique étrangère commune » depuis 1993 et, en raison de sa capacité d'influence politique et économique dans certains dossiers internationaux, elle peut être qualifiée d'acteur international, mais le concept ne fait pas l'unanimité. Nous examinerons les différentes dimensions de la puissance normative européenne afin de mettre en évidence leurs limites en nous focalisant sur la crise nucléaire iranienne et les divergences opposant l'UE aux États-Unis de D. Trump.

\footnotetext{
${ }^{1}$ SANTANDER, S., VLASSIS, A. (2018), «L’UE, une puissance commerciale bousculée », Diplomatie, $\mathrm{n}^{\circ} 47$, p. 74.

2 DEVIN, G. (2013), Sociologie des relations internationales, Paris, La Découverte, p. 76.

${ }^{3}$ FIEDLER, R. (2018), « Iran and the European Union after the Nuclear Deal », CES Working Papers, Alexandru Ioan Cuza University of Iasi, Centre for European Studies, Iasi, vol. 10, Issue 3, p. 297.
} 
L'axe majeur auquel nous nous attèlerons sera de déterminer comment et dans quelle mesure le retrait des États-Unis de l'Accord nucléaire (JCPOA, 2018) et l'imposition de la loi extraterritoriale américaine aux entreprises européennes ont révélé la limite et la faiblesse de la puissance de l'UE vis-à-vis des États-Unis. Cette question centrale peut se diviser en plusieurs sous-questions. Quelles sont les conséquences de l'arrivée au pouvoir de D. Trump sur les relations transatlantiques? Quelles sont les conditions du dialogue entre l'UE et l'Iran ? Pourquoi les États-Unis se sont retirés de l'Accord de 2015 ? Quels sont les effets de l'opposition de l'Arabie Saoudite et d'Israël à ce Traité sur la politique des États-Unis ? Pourquoi le projet nucléaire iranien a été perçu comme une menace par les États-Unis et l'UE ? À partir de ces questions, nous montrerons la différence des approches américaine et européenne au sujet de l'Iran. Notre travail se compose de trois parties. Dans la première, nous mobiliserons quelques concepts théoriques (puissance normative, soft power et acteur) afin de particulariser la puissance de l'UE. La deuxième partie se penchera, d'une part, sur la politique de l'UE à l'égard de l'Iran, et en particulier de son projet nucléaire, et d'autre part sur les négociations menées par le P5+1 avec l'Iran entre 2012 et 2015 (1'Accord multilatéral). Enfin, la troisième partie se focalisera sur le retrait états-unien de l'Accord de 2015 et sur les limites de la puissance européenne vis-à-vis de cette rétraction unilatérale.

\section{A) Les caractéristiques de la puissance de l'Union Européenne}

Pour comprendre les différents aspects de la puissance européenne, il importe de définir quelques concepts de sciences politiques relatifs à l'étude des Relations internationales. Il est nécessaire d'expliquer le concept de puissance (notamment le soft power), celui de puissance normative ainsi que celui dit « de l'acteur ». Ces trois vecteurs sont importants pour notre étude dans la mesure où l'UE est souvent qualifiée d'acteur international doté de puissance douce (soft power) ou de puissance normative, notamment par Ian Manners ${ }^{1}$.

\section{1) Le concept de puissance et sa typologie}

Le concept de puissance est évolutif, multidimensionnel et complexe. Sa définition varie en fonction des différentes approches des Relations internationales. Les réalistes mettent l'accent sur les aspects matériels, ou tangibles, de la puissance tandis que d'autres approches évoquent aussi les aspects immatériels comme la puissance structurelle et la puissance douce. Selon le penseur réaliste Hans J. Morgenthau, la politique internationale s'apparente à une lutte pour le pouvoir et la puissance se définit comme «l'emprise d'un homme sur les esprits et les actions des autres $»^{2}$. En raison du caractère anarchique de la société internationale, les États essayent de maximiser leur puissance et c'est pourquoi " [ils] cherchent à acquérir toute la puissance qu'ils peuvent obtenir $»^{3}$. Par ailleurs, d'après le fondateur du réalisme offensif, John Mearsheimer, : «ce que la monnaie est à l'économie, la puissance l'est pour les relations internationales ${ }^{4}$. Raymond Aron définit la puissance comme «la capacité d'une entité politique d'imposer sa volonté aux autres unités $»^{5}$. La puissance de l'État produit des aspects tangibles et non tangibles, et chaque dimension de la puissance revêt ses propres attributs. Joseph Nye, l'un des théoriciens de l'interdépendance complexe, a contribué à l'évolution du concept en introduisant une nouvelle typologie : la puissance douce (soft power) et la puissance dure (hard power). D'après lui, le soft power représente un pouvoir de séduction, un pouvoir de cooptation qui repose sur des ressources intangibles comme la culture, les institutions, les

\footnotetext{
${ }^{1}$ MANNERS, I. (2002), « Normative Power Europe: A contradiction in Terms? », Journal of Common Market Studies, vol. 40, n $^{\circ}$ 2, p. 236.

${ }^{2}$ MORGENTHAU, H. (1948), Politics Among Nations. The struggle for Power and Peace, New York, Knopf, p. 13.

${ }^{3}$ MEARSHEIMER, J. (2014), The Tragedy of Great Power Politics, USA, University of Chicago, p. 22.

${ }^{4}$ Ibid., p. 12.

${ }^{5}$ ARON, R. (1962), Paix et guerre entre les nations, Paris, Calmann-Lévy, pp. 16-17.
} 
idées... Il permet à son détenteur d'inciter les autres acteurs à l'imiter ${ }^{1}$. Le soft power accorde à l'État qui le détient la possibilité de « structurer une situation de telle sorte que les autres pays fassent des choix ou définissent des intérêts qui s'accordent avec les siens $»^{2}$. Au départ de cette définition du soft power, Nye met l'accent sur les nouvelles formes d'influence dans les Relations internationales à la fin de la guerre froide, et en particulier dans la pratique de la politique étrangère états-unienne.

\section{2) La puissance douce (soft power) et la puissance normative de l'Union Européenne}

En ce qui concerne le soft power de l'UE, il peut être défini comme tout ce qui lui permet de séduire, d'attirer et d'influencer le monde extérieur, sans recourir à la coercition ${ }^{3}$. On distingue deux formes de soft power: le «moderne» lié à la modernité politique et le « traditionnel ». Le soft power de l'UE est de type moderne et a une vocation universelle. Les différentes dimensions de la puissance douce de l'UE se manifestent par l'adoption de principes basiques : le respect des droits de l'Homme, l'État de droit, les droits des minorités, le modèle politique démocratique, le modèle de la paix démocratique par le biais du marché à travers une politique d'intégration, etc. Ces différentes valeurs constituent les facteurs de séduction et établissent la capacité d'influence de l'UE dans une partie de la communauté internationale, en particulier dans les sociétés civiles et auprès d'une partie des élites au Moyen-Orient dont les iraniennes. Dans ce cadre, nous pouvons également citer le cas des pays candidats à l'adhésion européenne. Dans les Relations internationales actuelles, on observe que les États tentent de mettre en œuvre une stratégie de soft power qui, depuis 1991, joue un rôle important dans la politique internationale.

Comme nous l'avons souligné supra, l'UE est qualifiée de puissance normative par une partie des chercheurs et, pendant la guerre froide, François Duchêne avait qualifié la Communauté européenne en termes de puissance civile (civilian power $)^{4}$. Mais ce concept a été critiqué par différents chercheurs, notamment par Hedley Bull ${ }^{5}$. La notion de puissance normative a été élaborée par Ian Manners pour qui l'UE demeure une puissance normative fondée sur un socle solide de valeurs, de normes et d'une identité, européenne et supranationale, qui oriente sa politique étrangère ${ }^{6}$. La puissance normative de l'UE comprend plusieurs aspects qui contribuent à sa diffusion dans les Relations internationales. Ian Manners en mentionne six : la contagion (contagion), la diffusion informationnelle (informational), la diffusion procédurale (procedural), le transfert (transference), la diffusion manifeste (overt diffusion) et le filtre culturel (cultural filter). Le premier (la contagion) se traduit par la diffusion de normes, de manière non intentionnelle, dont la substance ou l'idée est reprise et imitée par d'autres pays ou régions du monde à l'instar du Mercosur. La deuxième (la diffusion informationnelle) s'effectue via des communications stratégiques par des initiatives et des déclarations produites par les instances européennes. La troisième (la diffusion procédurale) se réalise dans une relation institutionnalisée entre l'UE et une tierce partie, à l'image de l'élargissement de l'UE vers des pays candidats à l'adhésion au projet européen. La quatrième (le transfert) se déploie lorsque Bruxelles échange des biens et accorde de l'aide ou de l'assistance technique à des pays tiers par le biais de moyens essentiellement matériels ou financiers, en exportant les standards

\footnotetext{
1 PAQUIN, S. (2009), "L'économie politique internationale et la mondialisation », dans PAQUIN, S. et DESCHÈNES, D. (dir.), Introduction aux relations internationales. Théories, pratiques et enjeux, Montréal, Chenelière, p. 37.

${ }^{2}$ NYE, J. (1990), Bound to lead. The Changing Nature of American Power, New York, Basic Books Publishers, p. 173.

${ }^{3}$ LEFEBVERE, M. (2011), La politique étrangère européenne, Paris, PUF, p. 68.

${ }^{4}$ DUCHENE, F, « Europe's role in world peace », in Richard Mayen (ed.), Europe tomorrow. Sixteen Europeans look ahead, London, Collins, 1972, p.35.

${ }^{5}$ PETITEVILLE, F. (2002), «L'Union européenne, un acteur international 'global' », Revue internationale et stratégique, $\mathrm{n}^{\circ} 43$, pp. 145-157.

${ }^{6}$ MANNERS, I. (2002), op. cit., p. 252.
} 
communautaires et en recourant à la politique « de la carotte et du bâton », soit par des récompenses financières ou des sanctions économiques, comme par exemple dans le cadre du programme d'aide communautaire aux pays d'Europe centrale et orientale (PHARE). La cinquième (la diffusion manifeste) se produit par le biais d'une présence physique de l'UE dans les pays tiers à travers des délégations de la Commission et des ambassades des États membres. Enfin, la sixième et dernière (le filtre culturel) affecte, à travers l'interaction, les normes internationales et l'apprentissage politique dans les pays tiers. La diffusion des droits de l'Homme en Turquie par exemple ${ }^{1}$ illustre cette expression normative qui, on doit le souligner, rencontre des difficultés de diffusion dans des pays comme la Chine ${ }^{2}$, la Russie, l'Iran, etc.

Zaki Laïdi, un autre auteur à avoir qualifié l'UE de puissance normative internationale, estime que « [1]a puissance renvoie à la force et la norme à la règle. La puissance appelle la puissance. Elle s'inscrit dans une dynamique de force ne reconnaissant ainsi que les limites qu'elle se fixe elle-même. À l'inverse, la règle renvoie à la contrainte, à la limite qu'il convient de fixer, à l'ordre qu'il faut préserver, aux frontières qu'il importe de ne pas dépasser. Pourtant norme et puissance se rejoignent sur un point essentiel : elles cherchent toutes les deux à contraindre $»^{3}$.

Depuis la fondation de l'UE, les normes ont joué un rôle déterminant dans sa transformation en espace de paix démocratique : « [1]a préférence de l'Europe pour la norme tient fondamentalement à l'essence même du projet européen depuis 1957. L'Europe cherche à dépasser les traditionnels conflits entre États en recourant au principe de souveraineté partagée sans pour autant abolir la souveraineté de ces mêmes États. Dans ces conditions, le seul moyen de lier durablement le destin de ces États, c'est de les faire adhérer à une norme commune d'autant plus contraignante qu'elle aura été négociée ${ }^{4}$. Pour Zaki Laïdi, la puissance normative de l'UE est composée à la fois des «normes politiques» et des «normes de marché », les secondes occupant un rôle primordial : «la puissance normative de l'Europe découle largement de son pouvoir de marché, mais l'impact de celui-ci dépasse le simple cadre du marché $»^{5}$. Selon l'auteur, il existe un lien entre la limite d'influence de la puissance normative de l'UE et les rapports de forces internationaux. Étant donné qu'elle ne dispose pas de toutes les fonctions régaliennes d'un État, « elle montre la limite de sa puissance dans la politique internationale en raison de l'absence d'une force militaire capable de défendre ses normes et d'une vision du monde relativement cohérente ${ }^{6}$. À travers cette étude, nous constatons quelques similitudes entre le soft power européen et une partie de sa puissance normative. Mais l'application des normes, liées à la puissance normative dans les relations internationales de l'UE, transite par des règles contraignantes. Cet examen préalable du concept de puissance (et sa typologie exprimée en puissances douce et normative), nous permet à présent d'expliquer la signification de l'acteur afin de répondre à la question : l'UE est-elle un acteur dans les Relations internationales, et en particulier dans le dossier nucléaire iranien ?

\section{3) Le concept de l'acteur et ses caractéristiques}

Dans la société internationale classique l'État est considéré comme le seul acteur des Relations internationales. Mais celles-ci ont évolué et d'autres composantes ont émergé et relativisé la souveraineté étatique. Il s'agit, notamment, selon James Rosenau, des acteurs

\footnotetext{
${ }^{1}$ Ibid., pp. 244-245.

${ }^{2}$ CABESTAN, J-P. (2015), La politique internationale de la Chine, Paris, Sciences Po, pp. 454-456.

3 LAÏDI, Z. (2009), «L'Europe, puissance normative internationale », dans DEHOUSSE, R., Politiques européennes, Paris, Presse de Sciences Po, p. 227.

${ }^{4}$ Ibid., p. 229.

${ }^{5}$ Ibid., p. 230.

${ }^{6}$ Ibid., p. 241
} 
«hors souveraineté ${ }^{1}$. L'histoire des Relations internationales montre que, parfois, ces éléments peuvent se muer en acteurs étatiques suite à un changement systémique comme une révolution ou l'arrivée d'un régime politique soutenu par des forces étrangères, à l'exemple des partis politiques kurdes en Irak après la chute de Saddam Hussein.

Le concept d'acteur est malaisé à cerner étant donné la multitude de définitions qui le caractérisent et l'absence d'unanimité parmi les spécialistes, en particulier dans le cadre de l'UE. Nous pouvons le définir comme une entité politique nantie de la capacité d'influencer le comportement d'une autre entité politique, étatique ou non-étatique. Cette capacité présente un caractère multiforme et comprend les aspects politique, militaire, culturel, économique et idéologique. Par ailleurs, la capacité d'influence peut prendre une dimension régionale ou mondiale selon le niveau de puissance de l'acteur concerné. Selon le dictionnaire des relations internationales, l'acteur peut être défini : «comme une entité dont les actions dans le champ international ou transnational ont un impact sur la distribution des ressources et sur la définition de certaines valeurs au niveau global $»^{2}$. Une étude, réalisée par Marcel Merle dans les années 1980, souligne les trois caractéristiques de l'acteur dans les Relations internationales : «par acteur, écrit-il, il faut entendre toute autorité, tout organisme, tout groupe et même, à la limite toute personne susceptible de jouer un rôle dans le champ social, en l'espèce sur la scène internationale $»^{3}$. Selon Merle, il se distingue par trois traits : «autorité, autonomie, et cohérence $»^{4}$.

D'autres chercheurs ont contribué à faire évoluer la notion d'acteur. Parmi eux, Joseph Jupille et James Caporaso ont ajouté une quatrième dimension au statut : « [1]a reconnaissance, en d'autres termes le fait que les autres acteurs acceptent d'interagir avec l'acteur concerné $»^{5}$. Cette reconnaissance contribue au façonnement de la personnalité juridique de l'acteur dans les Relations internationales. À partir de ces quatre critères, le statut d'acteur de l'UE dans les relations internationales peut être considéré à travers son rôle dans la politique internationale. Il faut aussi savoir que l'UE est dotée d'une personnalité juridique depuis le traité de Lisbonne et que cette particularité a renforcé son rôle international. Dans notre étude, nous appliquerons ces concepts aux cas de l'UE, de l'Iran et des États-Unis.

L'UE est une organisation régionale fondée sur des valeurs universelles. Depuis sa création, la politique d'intégration européenne a contribué à l'instauration de la paix et de la sécurité entre ses membres. Le processus d'élargissement de l'UE montre que celle-ci conditionne l'adhésion des nouveaux membres au respect de certaines exigences, en particulier les critères de Copenhague. Par le biais de ses critères et de ses normes, l'UE est en mesure de contribuer à la démocratisation de certains pays, il en a été ainsi pour les pays d'Europe centrale et orientale (Peco). Dans le cas de la Turquie, son adhésion est conditionnée à la réalisation de telles réformes. L'UE demeure aussi un acteur de médiation, de réconciliation et de paix (peacemaker) entre certains pays qui veulent devenir membre de l'UE : c'est le cas entre le Kosovo et la Serbie ${ }^{6}$.

\footnotetext{
${ }^{1}$ ROSENEAU, J. (1990), Turbulence in World Politics. A Theory of Change and Continuity, Princeton, Princeton University Press, p. 36.

2 BATTISTELlA, D., SMOUT, M-C., VENNESSON, P., PETITEVILlE, F. (2006), Dictionnaire des relations internationales, Paris, Dalloz, pp. 1-2.

${ }^{3}$ MERLE, M. (1988), Sociologie des relations internationales, Paris, Dalloz, p. 317.

${ }^{4}$ Ibid., p. 318.

5 JUPILlE, J. and CAPORASO, J. (1998), «States, Agency and Rules: The European Union in Global Environmental Politics » in RHODES, C. (ed.), The European Union in the World Community, Boulder, Colorado, Lynne Rienner, p. 214.

6 LIKA, L. (2016), «La pénétration turque dans les Balkans occidentaux: quels défis pour le projet d'élargissement de l'UE ? », dans SANTANDER, S. (dir.), Concurrences régionales dans un monde multipolaire émergent, Bruxelles, P.I.E. Peter Lang, pp. 250-251.
} 
Dans le cas du dialogue critique entre l'Iran et l'UE, celle-ci a conditionné ce dialogue au respect de cinq conditions. Elle a tenté de contraindre l'Iran à accepter ses normes dans la mesure où Téhéran avait besoin de l'UE dans un contexte marqué par des tensions avec les États-Unis. La capacité d'influence de l'UE dépend des rapports de force ; elle peut parfois imposer ses conditions aux acteurs étatiques ou non-étatiques qui sont moins forts qu'elle. Par ailleurs, quand l'UE présente des convergences avec de grandes puissances, elle peut se muer en acteur majeur dans la résolution de crise, à l'instar du rôle qu'elle a joué dans la conclusion de l'Accord nucléaire de 2015 entre l'Iran et le P5+1 et où elle a pu démontrer sa capacité d'influence diplomatique sur les autres acteurs.

L'évolution des relations internationales, en particulier le décentrage ${ }^{1}$ de la puissance dans le nouveau contexte mondial, affecte la puissance européenne. L'exemple des puissances émergentes, notamment la Chine $^{2}$ et la Russie, illustre parfaitement la limite de la puissance normative de l'UE. Durant les quelques années qui ont succédé à 1991, l'UE disposait d'une capacité d'influence politique et diplomatique à l'endroit de la Russie, mais l'arrivée au pouvoir de Poutine et la montée en puissance de la Russie ont changé la donne : «Il y a longtemps que l'UE ne sait pas bien quoi faire avec la Russie postsoviétique, malgré l'élaboration de divers partenariats économiques et stratégiques au cours des années 1990. Quant à la crise en Ukraine, déclenchée en 2013, elle traduit de manière plus spectaculaire encore les limites de la puissance 'civile' et 'normative' de l'UE $»^{3}$. À partir de ces observations théoriques et conceptuelles, nous allons à présent examiner les différentes étapes des relations entre l'UE et l'Iran, et en particulier les enjeux de la crise nucléaire iranienne.

\section{B) La Politique de l'Union Européenne à l'égard de l'Iran depuis 1992 jusqu'à nos jours}

En 1991, les Relations internationales sont entrées dans une nouvelle période caractérisée par l'hégémonie sans rival des États-Unis, même si cette hégémonie a pu être contestée $^{4}$ et affaiblie quelques années plus tard. Avec la fin du monde bipolaire, les dirigeants européens, sous l'égide de François Mitterrand et d'Helmut Kohl, ont relancé le projet de construction de l'Europe politique. L'un des objectifs de ce projet a été de contribuer à l'émergence d'un monde multipolaire dans lequel l'UE devait jouer un rôle important, un monde fondé sur l'équilibre des forces afin de limiter le pouvoir des États-Unis. Dans ce cadre, le Moyen-Orient constitue l'une des zones stratégiques où se joue la rivalité politique et économique entre l'UE et les États-Unis. Les divergences de Donald Trump et de l'UE sur la question iranienne résultent, en partie, de cette compétition dans la mesure où les États-Unis entendent affaiblir la puissance commerciale de l'UE.

\section{1) Les relations entre l'Union Européenne et l'Iran de 1992 à 1997 : le dialogue critique, un test pour la puissance normative de l'Union Européenne}

La politique de l'UE à l'égard de l'Iran s'inscrit dans le cadre de sa politique moyenorientale. Ses objectifs peuvent être décrits ainsi : favoriser les intérêts de l'UE par des relations commerciales et économiques, contribuer à la sécurité de la région riche en réserves de pétrole, contribuer à la paix régionale, promouvoir le respect des droits de l'Homme, et lutter contre la prolifération nucléaire. En effet, ces principes sont conformes aux objectifs de la politique

\footnotetext{
${ }^{1}$ SANTANDER, S. (2016) (dir.), Concurrences régionales dans un monde multipolaire émergent, Bruxelles, P.I.E. Peter Lang, p. 15.

${ }^{2}$ WANG, Y. (2009), «The Identity Dilemmas of EU Normative Power: Observations from Chinese Traditional Culture », in GERRITS André (ed.), Normative Power Europe in a Changing World: A Discussion, The Hague, Netherlands, Clingendael European Papers $n^{\circ}$ 5, pp. 73-74.

${ }^{3}$ PETITEVILLE, F. (2016), «L'Union européenne, acteur conditionnel de la résolution des conflits », dans BAZIN, A. et TENENBAUM, C. (dir.), L'Union européenne et la paix, Paris, Presses de Sciences Po, p. 129.

${ }^{4}$ BADIE, B. (2019), L'Hégémonie contestée. Les nouvelles formes de domination internationale, Paris, Odile Jacob, p. 9.
} 
étrangère commune européenne par le biais desquels l'UE s'emploie à diffuser ses normes sur le plan international; en l'occurrence dans ses relations avec l' $\operatorname{Iran}^{1}$. Mais la puissance normative européenne montre ses limites dans ces rapports étant donné que le régime iranien de nature théocratique (République islamique) ne reconnait pas l'universalisme des normes européennes.

Depuis 1992, la politique de l'UE envers l'Iran s'est poursuivie dans le cadre d'un « dialogue critique », suivi d'un « dialogue global » (constructif), dans le cadre duquel s'est tenue une réunion semestrielle entre une troïka européenne et les responsables iraniens. À l'origine, en 1992, les douze chefs des États membres de l'UE se sont réunis en Conseil à Edimbourg afin de déterminer la politique à suivre à l'égard du gouvernement iranien qui voulait développer ses relations avec l'UE. Le conseil Edimbourg généra : «(..) une stratégie de dialogue qui pos[a] le principe d'une concertation continue et cohérente avec l'Iran en vue d'inviter ce dernier à un comportement constructif sur des questions fondamentales pour l'Europe $»^{2}$. La demande de l'Iran s'inscrivait dans une double dimension. D'une part, au lendemain de la guerre avec l'Irak, sa situation socio-économique et politique se trouvait dans un état critique et le gouvernement de Rafsandjani n'avait d'autre choix que de redéfinir sa politique étrangère à travers une approche pragmatique. D'autre part, en raison de l'hostilité entre Téhéran et Washington, l'UE était la seule voie disponible pour rompre l'isolement et sortir de la crise économique.

Il est important de rappeler qu'un intérêt réciproque conditionnait les relations euroiraniennes. Soulignons de même que l'Iran est entouré de quinze pays, dont une partie possède de grandes réserves d'hydrocarbures, et qu'il se situe sur le chemin le plus direct pour l'acheminement vers l'Europe du pétrole de pays comme l'Azerbaïdjan. De son côté, l'Iran dispose de grandes réserves pétrolières et gazières (les deuxièmes plus importantes du monde). Au demeurant, avec 80 millions de citoyens qui composent sa population, il représente un grand marché avec des débouchés régionaux substantiels pour l'économie européenne. Selon la Commission européenne «[d]es raisons politiques et économiques se trouvent à la base de l'intérêt de l'UE pour l'Iran. Avec une position géographique stratégique et une importante réserve de gaz et de pétrole, ce pays pourrait jouer un rôle clé dans l'avenir de la région du Golfe. Selon la Commission, une démocratisation accrue et un plus grand respect des droits de 1'homme en Iran contribueraient à promouvoir la stabilité et la paix dans cette zone $»^{3}$.

En vertu de ces facteurs, l'UE a établi ses relations avec l'Iran dans le cadre du « dialogue critique » par lequel elle lui demandait les points suivants : « $1:$ respecter les droits de l'Homme en Iran ; 2 : renoncer au terrorisme et au soutien des mouvements intégristes ; 3 : non-prolifération des armes de destruction massive ; 4 : abolir la fatwa contre Salman Rushdie, écrivain britannique auteur des Versets sataniques ; 5 : cesser de s'opposer au processus de paix au Proche-Orient $»^{4}$. L'un des objectifs de l'UE par le dialogue critique a été d'influencer la politique de l'Iran sur deux plans. D'une part, elle lui demandait de procéder aux réformes sur les plans politique et économique; d'autre part, elle souhaitait une amélioration en ce qui concerne le respect des droits fondamentaux liés à la personne humaine. La réaction iranienne révéla deux approches. Comme l'explique A. V. Engeland-Nourai : « [1]e droit international des droits de l'homme n'a plus été la référence iranienne. Les Iraniens s'en sont remis au droit

\footnotetext{
${ }^{1}$ DUFAYS, N. (2012), «Les relations de l'Union européenne avec la République islamique de l'Iran : enjeux, perspectives et perceptions », dans MAKINSKY, M. (dir.), L'Iran et les grands acteurs régionaux et globaux, Paris, L'Harmattan, p. 432.

2 SCANDELLA, J. (2005), «L'Union européenne et l'Iran : entre institutionnalisation et suspension des relations », Les Cahiers de l'Orient, $\mathrm{n}^{\circ}$ 79, p. 105.

${ }^{3}$ Communication de la Commission au Conseil et au Parlement européen, Relations EU-République Islamique de l'Iran, le 7 février 2001.

${ }^{4}$ BAYRAMZADEH, K. (2004), Les enjeux principaux des relations entre l'Iran et l'Europe de 1979 à 2003 , Paris, l'Harmattan, p. 135.
} 
musulman pour définir la norme de référence des droits de l'homme. Toute dénonciation venue de l'Occident concernant la situation des droits fondamentaux était considérée comme une ingérence dans les affaires iraniennes $»^{1}$. Pendant le dialogue critique, l'UE et l'Organisation des Nations Unies (ONU) ont exercé une pression sur l'Iran à ce sujet et, dans un rapport sur la violation de ces droits daté de mars 1995, le gouvernement iranien fut mis en cause : «La commission des droits de l'homme de l'ONU a prolongé le mandat de son représentant pour une autre année. En août de la même année, la sous-commission des droits de l'Homme a condamné le gouvernement iranien pour la discrimination à l'égard des minorités ethniques en Iran, et les exécutions sommaires $»^{2}$.

Dans le cadre du « dialogue critique », l'UE a également enjoint l'Iran de mettre fin au soutien des mouvements islamistes, accusés de terrorisme par l'UE et les États-Unis, bien que selon le point de vue de Téhéran il s'agissait de mouvements de résistance à Israël. Soulignons d'ailleurs qu'à ce propos, l'Iran, sous la présidence de Rafsandjani, s'est opposé au processus de paix alors que le principal mouvement palestinien (Al-Fath) avait accepté la paix avec Israël. Cette posture iranienne provoqua la colère de l'UE, des États-Unis ainsi que des pays arabes. La troisième condition du dialogue critique tenait à la non-prolifération des armes de destruction massive et, dans ce contexte, l'UE maintenait la pression afin que de tels équipements ne soient pas développés. Mais du côté iranien, il s'agissait de pouvoir répondre à toute menace qui aurait pu surgir dans l'avenir ; particulièrement dans un contexte marqué par l'hostilité des États-Unis. L'Iran estimait n'avoir d'autre choix que de se fournir en avions, chars et missiles auprès de pays tels que la Russie, la Chine et la Corée du Nord. Une autre condition liée aux relations avec l'UE résidait dans la levée de la fatwa décrétée par Khomeyni à l'encontre de Salman Rushdie pour «blasphème » envers le prophète. L'Iran refusa et fit, à son tour, pression sur l'UE afin que les Versets sataniques soient interdits de publication et de diffusion mais celle-ci refusa.

Le dialogue critique n'a pas véritablement abouti et en 1997, suite au verdict du tribunal de Berlin, l'UE suspendit ses relations avec l'Iran. Selon le journal le Monde : «l'UE [s'était] montrée soucieuse de réitérer sa réprobation à l'égard des actions terroristes dont l'une, le meurtre de quatre opposants kurdes à Berlin, en 1992, [avait] été commandité, selon la justice allemande, par le haut sommet de l'État iranien. À cette fin, elle a confirmé solennellement la suspension du dialogue critique qu'elle entretenait depuis 1992 avec le régime des mollahs $»^{3}$. L'affaire de Mykonos, relative à l'assassinat de dirigeants du parti démocrate, entraîna une crise profonde entre l'UE et l'Iran. Ainsi, les gouvernements européens décidèrent de convoquer leurs ambassadeurs en Iran pour faire le point sur ce sujet et il en résulta une crise diplomatique qui dura sept mois.

À la lumière des explications relatives à cette première période des relations entre l'UE et l'Iran, nous constatons que l'UE a tenté de mettre en œuvre une politique commune et cohérente. Ce dialogue a permis à l'UE de tester une « politique étrangère commune » marquée par l'objectif central de développer des relations économiques et politiques avec l'Iran. Mais la réalité politique iranienne a révélé la limite de la puissance normative européenne. L'examen des cinq conditions montre que sur les plans commercial et économique, les relations ont connu une évolution importante caractérisées par une hausse de la présence des entreprises européennes en Iran et la croissance des échanges commerciaux entre les deux parties. Mais les autres conditions de l'UE n'ont pas abouti aux résultats escomptés. Cette difficulté a révélé que

\footnotetext{
${ }^{1}$ VAN ENGELAND-NOURAI, A. (2005), «Le rôle des droits de l'homme dans les relations entre l'Union européenne et l'Iran », Les Cahiers de l'Orient, ${ }^{\circ} 79$, p. 118.

${ }^{2}$ CORDESMAN, A. (1997), Iran: Dilemmas of dual containement, New York, Westview Press, p. 72.

${ }^{3}$ LEMAITRE, P. (1997), «L'Europe gèle le « dialogue critique » avec l'Iran mais ne prend pas de sanctions économiques », Le Monde, le 2 mai 1997, https://www.lemonde.fr/archives/article/1997/05/02/1-europe-gele-ledialogue-critique-avec-l-iran-mais-ne-prend-pas-de-sanctions-economiques 3540827_1819218.html (consulté le 15 janvier 2021).
} 
les normes européennes dans le cadre du dialogue critique s'opposaient aux valeurs politiques, culturelles et juridiques du régime iranien. En conséquence, l'UE n'a pas réussi à influencer le comportement politique de l'Iran et ce trait a constitué un défi pour la diplomatie européenne.

\section{Deux approches différentes entre l'Union Européenne et les États-Unis sur l'Iran pendant le dialogue critique}

Au contraire des Européens qui ont opté pour un dialogue politique et une coopération économique avec l'Iran, les États-Unis ont choisi une attitude d'isolement et de boycott dans le cadre d'une politique de « double endiguement » qui visait à la fois les gouvernements iranien et irakien. Cela correspond à une différence de visions entre l'UE et les États-Unis. L'UE entretient une approche libérale des relations internationales, basée sur le respect du droit international, le respect des droits fondamentaux, la négociation, la coopération économique et commerciale. Les États-Unis défendent une conception néo-réaliste, fondée sur la maximisation de l'intérêt national, leur sécurité et leur puissance : «Il est temps, écrit Robert Kagan, de cesser de faire comme si Européens et Américains partageaient la même vision du monde, ou même comme s'ils vivaient sur la même planète. L'Europe est en train de renoncer à la puissance ou, pour dire la chose autrement, elle s'en détourne au bénéfice d'un monde clos fait de lois et de règles, de négociation et de coopération transnationale. Elle pénètre dans un paradis post historique de paix et de relative prospérité, concrétisation de ce qu'Emmanuel Kant nomme la 'paix éternelle'. De leur côté, les États-Unis restent prisonniers de l'histoire, exerçant leur puissance dans le monde anarchique décrit par Hobbes ${ }^{1}$. Selon l'approche constructiviste, il existe trois sortes de cultures anarchiques ${ }^{2}$, l'UE se réclame de la culture anarchique kantienne, alors que les États-Unis relèvent de la culture anarchique hobbesienne.

L'objectif principal du «double endiguement » consistait à exercer une pression sur l'Iran afin qu'il renonce à son hostilité à l'égard d'Israël, au terrorisme et à son opposition au processus de paix : « [1] a politique de l'administration Clinton au sujet de l'Iran, entre 19931995, peut se résumer dans la poursuite d'une politique de double endiguement destinée à affaiblir les États iranien et irakien $»^{3}$. Cette politique fut complétée par un autre embargo économique et, en 1996, par la loi d'Amato qui prévoyait des sanctions contre toutes les compagnies pétrolières, même non-américaines, qui investiraient au-delà de 40 millions de dollars par an dans les deux pays, qualifiés d'États voyous par l'Amérique : l'Iran et la Libye. La portée extraterritoriale de cette loi a provoqué l'opposition unanime des pays de l'UE : «L'Europe reste fermement opposée à la législation des États-Unis qui a des effets extraterritoriaux, notamment à la loi d'Amato. Une telle législation est contraire au droit international $»^{4}$. Durant le dialogue critique, les Européens se sont démarqués des États-Unis en résistant à leur politique hégémonique, malgré la pression de ces derniers qui accusaient l'UE de faiblesse envers l'Iran, l'UE n'a pas cessé de poursuivre une politique indépendante.

2) L'Union Européenne et l'Iran pendant la présidence de Khatami (1997-2005) : une période marquée par une amélioration des rapports entre ces deux acteurs et par un demisuccès pour la puissance normative de l'UE

La victoire de Mohammad Khatami à l'élection présidentielle de 1997 a contribué à une amélioration relative des rapports irano-européens, dans la mesure où le nouveau président s'est démarqué des conservateurs du régime en appliquant une politique d'ouverture dans les

\footnotetext{
${ }^{1}$ KAGAN, R. (2004), La puissance et la faiblesse : Les États-Unis et l'Europe dans le nouvel ordre mondial, Paris, éd. Hachette, coll. Pluriel, p. 9.

${ }^{2}$ BATTISTELLA, D. (2012), Théories des relations internationales, Paris, Sciences Po, p. 315.

${ }^{3}$ KEMP, G. (1998), « The United States, Iran and Irak: Containement or Engagement? », US Policy and Iran, Paris, Edition Ifri, p. 32.

${ }^{4}$ La déclaration de sir Leon Brittan, le 30 septembre 1997, Bruxelles, Source d'Europe, 31494.
} 
relations extérieures, notamment envers l'UE. Depuis son arrivée au pouvoir, une page de la diplomatie iranienne à l'égard de l'UE a été tournée. La volonté de sortir de la crise dans laquelle se trouvaient les deux acteurs était réciproque, mais l'Iran ne disposait pas d'autre choix que de mettre fin à ses contentieux avec l'UE pour régler des problèmes importants sur le plan interne, qui étaient insolubles sans une ouverture de la politique étrangère. Quelques mois après l'arrivée au pouvoir de Khatami, l'UE a révisé sa position en adoptant une attitude positive à l'égard du nouveau gouvernement. Ce changement était lié à la déclaration du président iranien qui voulait donner de lui une nouvelle image, différente de celle de ses prédécesseurs, à travers son discours sur le respect de la liberté, la restauration de la société civile ainsi qu'une politique étrangère fondée sur la détente, le dialogue avec l'Occident ${ }^{1}$ et le « dialogue des civilisations ». Un ministre Britannique, Robin Cook, déclara ainsi sur le sujet : « Nous sommes prêts à aider les premiers pas de la glasnost en Iran. Nous nous félicitons des signes émis par le président iranien qui vont dans le sens d'une modernisation de l'économie et de la société ».

Après la reprise du processus de normalisation euro-iranien en 1998, le dialogue critique céda la place au dialogue global (constructif) qui abordait aussi bien les thèmes politiques que stratégiques. Il prit la forme de réunions semestrielles entre la troïka européenne et son homologue iranien. Le dialogue engageait des négociations dans trois domaines: 1) les questions régionales (Irak, golfe Persique, Asie centrale, processus de paix au Proche-Orient ; 2) les domaines de coopération (drogue, réfugiés, énergie, commerce et investissement) ; 3) les questions générales (terrorisme, droits humains et prolifération des armes de destruction massive). Dans ce contexte inédit, le nouveau gouvernement iranien renonça à l'application de la fatwa contre Salman Rushdie. Ce changement d'attitude fut un succès pour la diplomatie européenne dans la mesure où elle avait réussi, d'une part, à mettre en œuvre une politique cohérente et, d'autre part, à s'imposer comme acteur dans cette affaire. Cet exemple démontre que la puissance normative de l'UE peut rencontrer dans certaines conditions un écho favorable en fonction de l'identité des acteurs politiques.

\section{La stratégie de l'Union Européenne à l'égard de l'Iran durant les mandats de Khatami}

La stratégie de l'UE s'est concentrée sur le développement des rapports économiques et politiques, notamment, à partir de 2001-2002, coïncidant avec le deuxième mandat de Khatami. Cette stratégie prenait en compte l'importance de l'Iran sur le plan géopolitique, énergétique et économique dans un contexte politique marqué par la victoire des « réformistes » dans les élections législatives, augmentant ainsi le poids de Khatami vis-à-vis des «conservateurs ». Cette nouvelle donne politique suscita de nouveaux espoirs pour les Européens. Un rapport du Parlement européen expose cette stratégie : «A) Considérant que, au sens d'une stratégie de politique étrangère en matière de prévention des conflits, l'intérêt de l'UE réside dans un développement de l'Iran en un facteur de stabilité dans la région. B) Considérant que l'UE peut aider l'Iran à renouer des relations de bon voisinage avec tous les pays de la région et contribuer effectivement au développement de la coopération régionale. C) Considérant qu'avec un volume annuel d'exportation de plus de 4 milliards d'euros, l'UE présente le principal partenaire commercial de l'Iran $»^{2}$. En décembre 2002, à la suite de certains progrès, des négociations entre l'UE et l'Iran débutèrent en vue de conclure un Accord de commerce et de coopération. La Commission européenne lança des discussions en parallèle avec les négociations sur le dialogue politique et la lutte contre le terrorisme. Normalement elles auraient dû aboutir à un accord contractuel. Mais, l'UE a conditionné la conclusion de celui-ci au respect des droits humains ainsi qu'aux autres conditions précédemment abordées, et la question n'a

\footnotetext{
${ }^{1}$ FIEDLER, R. (2018), op. cit., p. 295.

${ }^{2}$ GAHLER, M. (2018), Le rapport du parlement européen sur les relations entre l'UE et la République islamique, Bruxelles, le 26 novembre 2001, pp. 6-8.
} 
pas manqué de diviser l'UE. Certains considéraient qu'il fallait séparer le volet économique du volet politique, alors que d'autres estimaient les deux volets indissociables. Ce qui ne manqua pas de montrer à quel point la cohésion au sein des pays de l'UE était faible.

En dépit d'une ouverture relative dans les relations euro-iraniennes depuis l'arrivée au pouvoir de Khatami, la révélation des sites nucléaires iraniens entraina une crise importante qui influença le processus des négociations: «C'est dans cette atmosphère qu'un groupe d'opposants en exil, l'Organisation des Moudjahidin du Peuple d'Iran (OMPI), [révéla] en 2002 que l'Iran [était] en train de construire une usine d'enrichissement d'uranium par centrifugation près de la ville de Natanz ainsi qu'une usine de production d'eau lourde près de ville d'Arak, destinée à alimenter un réacteur à eau lourde dont le chantier [devait] s'ouvrir à proximité ${ }^{1}$. Depuis cet événement, le «dialogue nucléaire» est devenu prioritaire pour la troïka européenne, les autres dialogues étant subordonnés aux progrès de ces négociations relatives au nucléaire ${ }^{2}$.

Les négociations se déroulèrent de décembre 2002 à juin 2003, à Bruxelles et à Téhéran. En 2003, suite à la crise nucléaire, elles s'arrêtèrent provisoirement et, en 2005, les discussions relatives à l'Accord de Commerce et de Coopération (ACC) ont repris, mais une nouvelle fois, elles furent suspendues pour le même motif en automne de la même année. Les objectifs de l'ACC peuvent être décrits ainsi : l'établissement d'un régime contractuel pour gérer le commerce UE-Iran, suivant les règles de l'Organisation Mondiale du Commerce (OMC) ; soutien de l'Iran dans son adaptation aux règles de l'OMC ; développement d'une coopération plus étroite dans des domaines comme l'énergie, le transport, l'environnement, la culture, l'immigration et les réfugiés ; encouragement aux réformes, renforcement de l'État de droit et progression du respect des droits humains.

Durant le dialogue global, notamment après les attentats du 11 septembre 2001, la divergence entre l'UE et les États-Unis s'accentua. Si ces derniers optèrent pour le durcissement de leurs politiques à l'égard de l'Iran, plaçant celui-ci dans la liste des pays de « l'Axe du mal », l'UE choisit la voie du dialogue, en donnant la priorité au respect du droit international et à la négociation, afin de trouver une solution diplomatique aux problèmes, notamment à celui de la crise nucléaire. Cette attitude de l'UE fut assimilée à une stratégie de la carotte par opposition à celle du bâton, mais pour une partie des observateurs, la politique de l'UE à l'égard de Téhéran s'est révélée inefficace car par ces négociations, l'Iran a essayé de gagner du temps pour obtenir la technologie nucléaire. Néanmoins, il faut rappeler que, par la trö̈ka européenne et notamment par le rôle important joué par Javier Solana, Haut représentant pour la politique étrangère et de sécurité commune, l'UE a réussi à faire signer à l'Iran le protocole additionnel de l'Agence Internationale de l'Énergie Atomique (AIEA) le 29 novembre 2004. Mais la reprise de l'activité nucléaire de l'Iran en 2005 a de nouveau entraîné une crise importante dans les rapports euro-iraniens.

De ce fait, pendant les mandats de Khatami, malgré les progrès relatifs dans les relations entre l'UE et l'Iran en matière de politique étrangère et de coopération commerciale, la crise nucléaire a empêché la conclusion de l'ACC. Durant cette période, le président iranien s'est rendu dans plusieurs pays européens, notamment en France et en Allemagne, moteurs au sein de l'UE, et a, de la sorte, obtenu une certaine reconnaissance. Au cours de cette période, une partie des normes européennes a rencontré un écho favorable auprès des « réformateurs » du système iranien. En ce qui concerne la crise nucléaire, l'UE a utilisé sa capacité d'influence sur certains acteurs iraniens afin d'amener l'Iran à la signature du protocole additionnel de l'AIEA, le 29 novembre 2004. Mais il n'a pas été ratifié par le Parlement iranien. Cette situation a

\footnotetext{
${ }^{1}$ NICOULLAUD, F. (2016), «La fin d'un bras de fer avec la communauté internationale ? Retour sur douze années de négociations nucléaires », Iran le retour, Questions internationales, $\mathrm{n}^{\circ}$ 7, La documentation française, p. 22.

${ }^{2}$ DUFAYS, N. (2012), op. cit., p. 425.
} 
montré que le changement des acteurs politiques iraniens a modifié la position de l'UE et que, par conséquent, la capacité d'influence politique et économique de l'UE s'est accrue pendant quelques années.

3) Les rapports entre l'Union Européenne et l'Iran sous la présidence d'Ahmadinejad (2005-2013) : une période marquée par la dégradation des rapports entre l'UE et l'Iran et par le renforcement de la position régionale de l'Iran au Moyen-Orient

Depuis l'arrivée au pouvoir de Mahmoud Ahmadinejad en juin 2005, la politique étrangère de l'Iran a subi des changements importants non sans conséquences sur les rapports euro-iraniens. Sa nouvelle politique étrangère s'est caractérisée par quatre axes majeurs : une hostilité croissante à l'égard d'Israël, un anti-américanisme avéré, une volonté d'accroître sa puissance au Moyen-Orient et une velléité d'obtenir la technologie nucléaire en résistant à la pression des États-Unis et de l'UE. Cette politique s'est concrétisée par le contexte régional qui a contribué à la montée en puissance de l'Iran.

Depuis juin 2005, les rapports euro-iraniens se sont poursuivis dans le cadre des conditions précédentes, mais deux problèmes ont marqué ces relations. D'abord, la reprise des activités nucléaires iraniennes au début d'août 2005 : « [1]es Européens ont cherché à obtenir de l'Iran une renonciation à toute maîtrise du cycle du combustible nucléaire et une ratification du protocole additionnel du TNP en échange d'offre de coopération dans les domaines du nucléaire civil, de l'énergie et d'un engagement de l'UE à soutenir la candidature iranienne à l'OMC. La négociation a toutefois été compromise par la décision du nouveau président Ahmadinejad de relancer l'enrichissement d'uranium en août $2005 »^{1}$. Ensuite, la déclaration d'Ahmadinejad à l'encontre de l'État hébreu qui, selon le président iranien, devait être rayé de la carte. Cette attitude provocatrice a engendré une protestation de l'UE à l'égard de l'Iran.

\section{Les effets de la crise nucléaire sur l'avenir des relations entre l'Union Européenne et l'Iran}

La reprise des activités nucléaires iraniennes a entraîné une crise importante avec l'UE. Pour trouver une solution diplomatique à ce problème, les Européens ont, en août 2005, proposé à l'Iran une offre globale sur les questions nucléaires, commerciales et de sécurité régionale, mais elle fut qualifiée d'inacceptable et d'insultante et rejetée en tant que telle. Dès lors, l'Iran a remis en marche le processus de conversion dans l'usine d'Ispahan. En 2006, après l'échec des négociations prévues par le «package » du groupe P5+1, la visite de Kofi Annan à Téhéran (5 septembre 2006) et les rencontres de Javier Solana durant le mois de septembre avec son homologue Ali Laridjani, le numéro 2 du programme nucléaire iranien a présenté une contreproposition : créer un consortium mené par l'entreprise française Areva pour accompagner l'enrichissement d'uranium. Mais les Européens l'ont rejetée.

Le 23 décembre 2006, le Conseil de sécurité de l'ONU a adopté la résolution 1737 qui a donné un délai de deux mois à l'Iran pour suspendre son programme d'enrichissement d'uranium. Malgré cet ultimatum, Téhéran est passé outre et, depuis le début de 2007, l'UE a adopté les sanctions décrétées par la résolution 1737. Ces sanctions concernent essentiellement l'interdiction de transfert de technologie nucléaire et balistique, la restriction de déplacement de personnes liées au programme nucléaire et le gel des avoirs de ces dernières. En effet, l'objectif principal de la politique de sanction, relevant de la diplomatie coercitive, consistait à affaiblir le gouvernement iranien afin qu'il renonce à son projet nucléaire. En dépit des sanctions envisagées par le Conseil de sécurité et l'UE, l'Iran a poursuivi son programme d'enrichissement et Mahmoud Ahmadinejad insista sur le droit légitime de son pays à produire l'énergie nucléaire dans le cadre de l'article 4 du Traité sur la Non-prolifération des Armes

\footnotetext{
${ }^{1}$ PETITEVILlE, F. (2006), La politique internationale de l'Union européenne, Sciences Po, p. 57.
} 
Nucléaires (TNP) ${ }^{1}$. Les pourparlers entre le négociateur iranien (M. Djalili qui a remplacé A. Laridjani) et J. Solana en 2008 n'ont pas abouti à des solutions acceptables pour les deux parties. Selon le gouvernement iranien, l'UE fut influencée par les États-Unis et Israël dans sa démarche.

Dans cette situation, force fut de constater que, d'une part, les négociations entre l'UE et l'Iran et, d'autre part, entre l'Iran et l'AIEA ont été dans l'impasse dans la mesure où l'Iran n'a pas renoncé à son projet. En outre, le directeur de l'Organisation de l'énergie atomique d'Iran « M. Aghazadeh a confirmé en novembre 2008 que l'Iran avait installé cinq mille centrifugeuses à Natanze $»^{2}$. Ainsi, l'Iran a envoyé des signaux de fermeté aux Européens et aux Américains dans un contexte politique marqué par l'arrivée au pouvoir d'Obama qui a réitéré son opposition au projet nucléaire iranien tout en exprimant sa volonté de dialoguer avec les dirigeants iraniens ${ }^{3}$. Malgré l'échec des négociations, l'UE a rejeté l'option militaire pour régler le problème iranien, estimant qu'un recours armé embraserait la région et entraînerait une crise internationale majeure ${ }^{4}$. C'est pourquoi, en dépit de l'intensification de la politique de sanction depuis 2010 à l'égard de l'Iran par l'UE, celle-ci a toujours insisté sur le dialogue et la solution diplomatique.

Les événements du 11 septembre ont bouleversé les relations internationales. La politique étrangère américaine s'est durcie et la décision d'inclure l'Iran dans « l'Axe du mal » ${ }^{5}$ marqua la rupture des relations entre les deux pays. En 2002, Washington accusa l'Iran de financer les terroristes d'Al-Qaeda. Depuis l'intervention états-unienne en Irak, les rapports sont entrés dans une phase critique, marquée par l'accentuation des menaces de la part de Washington, et la tension n'a cessé de croître à travers les déclarations officielles. Depuis la découverte d'un site clandestin d'enrichissement d'uranium, les États-Unis ont demandé à l'ONU de prendre des sanctions contre l'Iran. La nouvelle donne politique, marquée par l'arrivée de Barak Obama à la présidence des États-Unis en 2009, a ouvert la voie à une nouvelle politique caractérisée par le respect et l'engagement diplomatique vis-à-vis de Téhéran. Le 20 mars 2009, l'administration Obama s'est déclarée «attachée à une diplomatie incluant l'ensemble des questions qui se [présentaient à elle], en vue de nouer des liens constructifs entre les États-Unis, l'Iran et la communauté internationale (...) ce processus n'avancera pas grâce aux menaces $»^{6}$. En effet durant l'année 2009, l'administration américaine s'est dit prête au développement d'une «politique de la main tendue» en affichant des signes d'assouplissement ${ }^{7}$; cette offre de conciliation a toutefois été rejetée par Téhéran la même année $^{8}$. Avec l'arrivée d'Ahmadinejad, et sa réélection en 2009, la position des États-Unis et de l'UE à l'égard de l'Iran a changé. Deux nouveaux problèmes majeurs sont apparus. D'une part la relance du projet nucléaire et, d'autre part, les déclarations ouvertement antisionistes du président. Dès lors, les positions européenne et américaine se sont progressivement rapprochées.

\footnotetext{
${ }^{1}$ ZARIFIAN, J. (2015), « Le dossier nucléaire iranien et la question du rapprochement États-Unis-Iran », Politique américaine, $\mathrm{n}^{\circ} 26$, p. 34.

${ }^{2}$ Mehr News (site web iranien) : www.mehrrnews.com/fa/ (26.11.2008).

${ }^{3}$ EIFFLING, V. (2013), «Les États-Unis face à la question nucléaire iranienne : un nœud gordien perpétuel ? », dans NAHAVANDI, F. (dir.), L'Iran dans le monde, Paris, l'Harmattan, p. 54.

${ }^{4}$ BONIFACE, P. (2019), Comprendre le monde, Paris, Armand Collin, p. 273.

${ }^{5}$ LAROCHE, J. (2011), La brutalisation du monde : du retrait des États à la décivilisation, Québec, Liber, pp. 7677.

${ }^{6}$ MICHEL, L. (2009), «La refonte de la stratégie de défense américaine », Revue Internationale et Stratégique, $\mathrm{n}^{\circ} 76, \mathrm{pp} .147-154$.

7 MIKHAÎL, B. (2010), «Une diplomatie du pragmatisme aux effets limités au Moyen-Orient?», Revue Internationale et Stratégique, $\mathrm{n}^{\circ} 77$, p. 145.

${ }^{8}$ LESNES, C. (2013), «La politique étrangère de Barack Obama : la tentation du repli ? », dans BADIE, B. et VIDAL, D. (dir.), Puissances d'hier et de demain, La Découverte, p. 101.
} 


\section{Le changement de stratégie de l'Union Européenne à l'égard de l'Iran depuis 2010}

Depuis juin 2010, l'UE a mis en œuvre une nouvelle politique à l'égard de l'Iran, concrétisée par une série de sanctions unilatérales relevant de la politique de l'arme économique : l'UE a fait pression sur le gouvernement iranien afin qu'il accepte ses conditions. Avant cette date, la politique de l'UE consistait à négocier dans le cadre d'un dialogue critique (de 1992-1997) et d'un dialogue global (depuis 1998) afin d'aboutir à la normalisation de leurs relations politiques et à la conclusion de l'ACC. En décembre 2002, la Commission européenne a lancé les négociations sur l'ACC, mais depuis 2005, elles sont suspendues en raison de la reprise des activités nucléaires iraniennes. Cet Accord présente une importance stratégique pour l'économie iranienne dans le sens où il devrait lui permettre d'accéder aux marchés européens et faciliter l'adhésion à l'OMC. Pour la première fois dans les rapports euro-iraniens, nous avons constaté une convergence entre l'UE et les États-Unis sur le dossier iranien alors que, dans le passé, leurs approches divergeaient sensiblement. Le changement de politique européenne s'explique par le choix du régime iranien en faveur d'un accès à l'énergie nucléaire mis en doute par l'UE quant à la vocation pacifique de ce projet. C'est pourquoi depuis 2010, elle a mis en œuvre des sanctions économiques afin d'exercer une pression sur le régime iranien et le ramener à la table des négociations, «L'Union européenne a décidé de ne plus acheter de pétrole iranien à partir de juillet $2012 »^{1}$. Les positions des États-Unis et de l'UE convergeaient donc sur la question iranienne alors que la Russie et la Chine n'avaient pas la même perception sur ce sujet.

La politique de la Russie à l'égard du projet iranien s'est démarquée des approches américaine et européenne, et ce pour plusieurs raisons. En 1995 la Russie s'est opposée à l'embargo décrété par Clinton et a accepté de construire la centrale nucléaire de Boucher. Les compagnies Statoil et surtout Gazprom commencèrent de leur côté à participer à des contrats d'exploitation du pétrole et du gaz iranien. À peine la première guerre du Golfe était-elle terminée que les Russes ont profité des possibilités de partenariat et depuis la coopération n'a cessé de se renforcer. Dans la crise du nucléaire, Moscou a d'abord privilégié la solidarité avec les États-Unis pour des raisons économiques et politiques évidentes mais à partir de 2006, Moscou s'est distingué dans son attitude face à Téhéran. La visite de Vladimir Poutine le 16 octobre 2007 a marqué un tournant majeur et a débouché sur plusieurs accords et sur la livraison de carburant nucléaire pour la centrale de Boucher. En 2008, la Russie a cessé de soutenir les sanctions contre Téhéran. Après les innombrables échecs d'accord entre les cinq membres permanents du Conseil de sécurité de l'ONU plus l'Allemagne («P5+1») et Ahmadinejad, l'Iran a été condamné par trois résolutions pour ses refus de suspendre son enrichissement en uranium et d'apporter des éclaircissements sur le caractère non militaire de ses activités passées et présentes. La résolution 1696 du 31 juillet 2006 enjoignait l'Iran de suspendre ses activités d'enrichissement et prévoyait, en cas de refus, d'envisager des sanctions qui autoriseraient des mesures économiques prévoyant un recours à la force, en accord avec l'article 47 du chapitre VII de la charte de l'ONU. Comme toujours, la Chine a insisté sur la solution diplomatique. Selon Qin Gang, le porte-parole du Ministère des Affaires étrangères de la Chine en 2010, c'est la manière la plus appropriée pour trouver une solution répondant aux préoccupations de toutes les parties. La Chine s'est opposée à toute sanction relative au pétrole dans la mesure où elle est fortement dépendante de pays producteurs tels que l'Iran dont elle est, par ailleurs, le premier partenaire commercial ${ }^{2}$.

Deux mois après la dernière résolution onusienne, et peu de temps après les États-Unis, l'UE a décidé unilatéralement de durcir les sanctions à l'encontre de l'Iran, à travers le secteur gazier et pétrolier, soit un domaine de haut enjeu stratégique pour l'Iran car, bien qu'il soit

${ }^{1}$ THERME, C. et KHAZANEH, R. (2012), « Le développement du programme nucléaire iranien : entre aspects techniques et questions politiques », Maghreb-Machrek, $\mathrm{n}^{\circ} 212$, p. 115.

${ }^{2}$ CABESTAN, J-P. (2015), op. cit., pp. 496-497. 
classé quatrième producteur mondial de brut, il devait importer $40 \%$ de son essence, faute de capacités de raffinage. Par conséquent, l'UE, sur les conseils de l'AIEA, a décidé d'attaquer son point faible en interdisant nouveaux investissements, transferts de technologie et assistance technique. En outre, les personnalités affiliées aux Gardiens de la révolution furent soumises à des sanctions individuelles. Ces mesures eurent pour effets immédiats la suspension complète des activités de Total (le 18 octobre 2010) et la fermeture d'un gisement gazier dans la mer du Nord, détenu à parts égales par la filiale britannique BP et la compagnie nationale du pétrole iranien (NIOC) $)^{1}$. De leurs côtés, la Russie et la Chine, considérées comme « (...) des alliés économiques de l'Iran (...) se sont prononcés hostiles à toute sanction dirigée contre le régime des mollahs $»^{2}$. La continuation de la politique de sanctions a eu des effets importants sur l'économie iranienne, et en partie a influencé la position de l'Iran dans ses négociations avec le groupe 5+1 pour aboutir à l'accord de Genève en 2013.

\section{4) L'Union Européenne et l'Iran depuis l'arrivée au pouvoir de Hassan Rohani en 2013 ; une période caractérisée par des négociations multilatérales entre l'Iran et le groupe P5+1 qui a abouti à l'Accord de 2015, et par le retrait des États-Unis de ce dernier en 2018}

En 2013, Hassan Rohani a été élu président de la République du régime iranien. Depuis son arrivée au pouvoir, le nouveau président a mis, d'une part, l'accent sur la résolution diplomatique et politique du dossier nucléaire iranien et, d'autre part, il a évité d'employer un discours radical à l'adresse des États-Unis et d'Israël. Par ailleurs, il a réaffirmé que le projet iranien n'avait pas une dimension militaire et a précisé que son pays était prêt à négocier avec la communauté internationale tout en réaffirmant le droit de l'Iran d'accéder au nucléaire civil. «En ce qui concerne la question épineuse de la diplomatie nucléaire, Hassan Rohani est un bon connaisseur du dossier. De ce fait, il pourra faire repartir les négociations $»^{3}$. Quant aux relations de l'Iran avec l'UE, il a mis l'accent sur la coopération et la négociation. L'arrivée au pouvoir de Hassan Rohani en 2013 a permis à l'UE de renforcer son rôle dans les négociations : " cette élection a permis au groupe de contact de rompre avec la politique d'intransigeance iranienne et entraîne le retour en force de la diplomatie européenne, dont les intérêts sont défendus par la Haute Représentante, Lady Ashton ${ }^{4} »$.

Dans un contexte régional marqué par la crise au Moyen-Orient, l'Iran a continué à soutenir le régime syrien, avec l'aide de la Russie et du Hezbollah. Cette intervention a empêché le renversement de Bachar el-Assad ${ }^{5}$ et a démontré le rôle majeur joué par l'Iran sur le plan régional. L'Iran a accepté de revenir à la table des négociations avec les grandes puissances et l'UE à partir de février 2013 et d'entrer dans une nouvelle période de discussions en vue de trouver une solution diplomatique avec le P5+1. Dans cette phase : «l'UE a repris possession du rôle de porte-parole et de conciliateur qui lui [avait] été attribué sous Solana ${ }^{6}$. Les tractations ont abouti, le 24 novembre 2013, à l'accord préliminaire de Genève qui fut un compromis entre l'Iran et le groupe P5+1, qui mit un terme à une crise de 10 ans : «La stratégie des membres du P5+1 a été de trouver dans un premier temps un accord provisoire pour servir

\footnotetext{
${ }^{1}$ Agence France Presse, le 26 octobre 2010.

2 BLACHER, P. (2006), Droit des relations internationales, Paris, Litec, p. 154.

3 DJALILI, M-R. (2013-2014), «Politique étrangère de la République islamique : le rôle du président », Confluences méditerranée, $\mathrm{n}^{\circ} 88$, Paris, l'Harmattan, p. 31.

${ }^{4}$ VIAUD, A. (2017), L'Union européenne face à la crise nucléaire iranienne, Belgique, Presses universitaires de Louvain, p. 127.

${ }^{5}$ BAYRAMZADEH, K. (2013), «La lutte pour l'hégémonie régionale dans les relations internationales : le cas du conflit syrien », Cahiers de Sciences politiques de l'ULG, Cahier n²7, disponible à l'adresse suivante : http://popups.ulg.ac.be/csp/ (consultée le 13 juillet 2020).

${ }^{6}$ VIAUD, A. (2017), op. cit., p. 128.
} 
de base de discussion commune $»^{1}$. Cet accord a fixé une période transitoire pour aboutir à une forme définitive, pour autant qu'un respect réciproque des engagements soit assuré.

Dans la continuité des négociations, le 14 juillet 2015, l'Iran et le P5+1 sont parvenus à signer un texte définitif qui a reconnu à l'Iran le droit d'utiliser l'énergie nucléaire de manière pacifique, à condition de respecter les engagements prévus par l'Accord. La conclusion du texte de Genève 2015 a contribué à l'amélioration des rapports entre l'Iran et l'UE. L'implication diplomatique de cette dernière dans le processus des négociations de 2003 à 2015 a favorisé son rôle d'acteur des relations internationales. Notre analyse démontre d'une part, la cohérence des pays membres de l'UE qui ont montré leur unité dans cette crise et, d'autre part, du rôle joué par le Haut représentant de l'UE pendant les différentes étapes des négociations.

Selon le texte de 2015 (JCPOA), l'Iran s'engage, pendant quinze ans, à ne pas enrichir l'uranium au-delà de $3.67 \%$ et à ne conserver sur son sol qu'au maximum 300 kilogrammes d'uranium légèrement enrichi. L'Iran ne pourra faire fonctionner plus de 5060 centrifugeuses de première génération pendant dix ans et s'engage à appliquer le protocole additionnel de l'AIEA ${ }^{2}$. Cet Accord a permis la levée des sanctions à partir du 16 janvier 2016. Progressivement, les entreprises européennes ont repris leurs activités et ont signé des contrats avec l'Iran : il s'agit notamment de Renault-Nissan, Airbus, Peugeot, Volkswagen, etc. Cet Accord a constitué un tournant dans la crise nucléaire iranienne bien que l'Arabie Saoudite et Israël s'y sont opposés. L'Arabie Saoudite, alliée stratégique des États-Unis au Moyen-Orient, a mis en cause Washington en lui reprochant de s'être rapproché de Téhéran au détriment de Ryad : « l'accord du 14 juillet aussitôt signé, quelques voix [se sont élevées] pour exprimer un vif mécontentement : le prince Bandar Bin Sultan a dénoncé ses conséquences sur le MoyenOrient $^{3}$ ». En ce qui concerne l'État hébreu, « c'est une erreur historique » déclara son Premier ministre, Benyamin Netanyahou. Les convergences entre ces deux pays étant liées à leur perception de la menace ${ }^{4}$ qui voit le projet nucléaire iranien comme un dilemme de sécurité. L'Arabie Saoudite et Israël ont exercé une pression sur les États-Unis afin qu'ils se retirent de l'Accord nucléaire et ont trouvé un écho favorable auprès du président Donald Trump qui réalisa ce vœu en 2018.

\section{C) Les impacts de l'arrivée au pouvoir de Donald Trump sur l'Accord nucléaire de 2015 (JCPOA) ; une période marquée par une dégradation des relations entre les États- Unis et l'Union Européenne}

Depuis l'arrivée au pouvoir de Donald Trump, la nouvelle politique des États-Unis se caractérise par l'isolationnisme, le retrait de plusieurs traités internationaux, en particulier l'Accord multilatéral portant sur le nucléaire iranien, le désengagement de plusieurs organisations internationales, l'unilatéralisme et le néomercantilisme. Cette nouvelle politique est en rupture avec celle d'Obama. Par ces différentes actions, "il rejette l'ordre multilatéral basé sur le compromis, la négociation, la coopération et le respect du droit international $»^{5}$. Selon Dario Battistella, « la vision mercantiliste est liée à l'approche réaliste de la politique internationale, synonyme d'état de guerre. En ce qui concerne le commerce, l'enrichissement n'est possible que si la balance extérieure d'un État est excédentaire, d'où des politiques commerciales multipliant les prohibitions et les barrières aux importations, les primes et les

\footnotetext{
${ }^{1}$ ROUSSELET, L. (2017), Négocier l'Atome : Les États-Unis et les négociations de l'accord sur le nucléaire iranien, Paris, l'Harmattan, p. 72.

${ }^{2}$ Voir le contenu complet de l'Accord dans l'article de NICOULLAUD, F. (2016), op. cit., pp. 28-29.

${ }^{3}$ MAKINSKY, M. (2016), «Iran-Arabie Saoudite : quel dialogue après l'accord nucléaire ?», Stratégique Orients, $\mathrm{n}^{\circ}$ 3, l'Harmattan, p. 33.

${ }^{4}$ LOISEAU, H. (2009), «Les approches contemporaines des relations internationales », dans PAQUIN, S. et DESCHENES, D. (dir.), Introduction aux relations internationales, Canada, Chenelière Education, p. 25.

${ }^{5}$ SANTANDER, S. et VLASSIS, A. (2020), «EU in Global Affairs: Constrained Ambition in an Unpredictable World? », European Foreign Affairs Review, vol. 25, nº 1, p. 15 (traduit par nos soins).
} 
subventions à l'exportation et les représailles à l'encontre des mesures prises par les autres États ${ }^{1} »$. La politique actuelle des États-Unis à l'égard de l'UE, de la Chine et de la Russie, est fortement liée à cette approche économique, et nous constatons la trace de cette vision dans différentes déclarations de D. Trump à l'adresse de la Russie, l'UE, l'Iran et la Chine.

\section{1) La stratégie de D. Trump à l'égard de l'Iran et ses conséquences sur l'Union Européenne}

Dans ce nouveau contexte, Donald Trump veut affaiblir la puissance commerciale de l'UE par l'imposition de sa loi extraterritoriale aux entreprises étrangères, y compris les entreprises européennes. Ces changements d'orientation de la politique étrangère des ÉtatsUnis contribuent à montrer que les relations internationales sont entrées dans une nouvelle période, marquée par l'affaiblissement de la puissance hégémonique des États-Unis et la transformation du rôle des pays comme la Chine et la Russie. Ces deux dernières sont devenues de grandes puissances et constituent un défi pour la puissance états-unienne. Selon J. Mearsheimer, «cette situation montre la grande difficulté de l'ordre international libéral et annonce son affaiblissement $»^{2}$. Pour comprendre la stratégie des États-Unis, il est crucial d'appréhender cette problématique à travers la transformation des rapports de forces régionales et mondiales. Les États-Unis s'opposent à la montée en force de l'Iran en tant que puissance régionale hégémonique au Moyen-Orient, en particulier dans le golfe Persique, car d'après les États-Unis, si l'Iran atteint cette position, le pays aura peut-être la possibilité de se projeter dans d'autres régions du monde. C'est pourquoi, «D. Trump a mis en place des politiques de la pression maximale sur l'Iran $»^{3}$. Selon Donald Trump, l'Accord de 2015 a favorisé l'Iran, et l'accès de celui-ci au stade « atomique » peut renforcer sa puissance hégémonique au MoyenOrient, ce qui est considéré comme une menace pour les intérêts stratégiques des États-Unis. Cette politique s'inscrit dans une stratégie globale qui consiste à empêcher que les puissances européennes et asiatiques se transforment en des puissances régionales hégémoniques ${ }^{4}$. Dans la perception de Donald Trump, l'Iran demeure un pays qui présente des menaces pour les alliés américains que sont l'Arabie Saoudite ${ }^{5}$ et Israël. De ce fait, le retrait des États-Unis de l'Accord de 2015 est lié à une politique multidimensionnelle mixant des aspects économiques, commerciaux, politiques, sécuritaires et stratégiques. Il faut aussi préciser que, depuis le retrait, l'Iran s'est rapproché de la Chine et de la Russie.

\section{2) La politique de l'Union Européenne face au retrait des États-Unis de l'Accord de 2015}

Pendant la période électorale, Donald Trump avait déjà annoncé que, sous sa présidence, les États-Unis se retireraient de l'Accord, et c'est ce qu'il a fait en 2018 en réinstaurant les sanctions contre l'Iran. Cette décision fut en contradiction avec les intérêts de l'UE et des pays signataires et souleva une vague de mécontentement et de protestation auprès des responsables de l'UE. Ces sanctions unilatérales américaines provoquèrent un contentieux international inédit auprès, notamment, des entreprises européennes ou chinoises ${ }^{6}$. L'UE a continué à défendre l'Accord et tenté de mettre en pratique des mesures commerciales permettant aux entreprises européennes de commercer avec l'Iran sans qu'elles soient sanctionnées par les

\footnotetext{
${ }^{1}$ BATTISTELlA, D. (2011), Paix et Guerres au XXI siècle, Paris, Sciences Humaines Editions, pp. 57-58.

${ }^{2}$ MEARSHEIMER, J. (2019), « Bound to Fail, The Rise and Fall of the Liberal International Order », Security, vol. 43, n 4, pp. 7-50 (traduit par nos soins).

${ }^{3}$ CIZEL, A. (2020), «L'obsession iranienne : les États-Unis au défi des (dès)équilibres régionaux », dans THERME, C. (dir), L'Iran et ses rivaux, Passés composés. p, 35.

${ }^{4}$ MEARSHEIMER, J. (2012), op. cit., pp. 40-42.

${ }^{5}$ KANDAL, M. (2018), «États-Unis-Arabie Saoudite, une alliance ambiguë », Questions internationales, Paris, La documentation française, $n^{\circ} 89$, pp. 82-83.

${ }^{6}$ HOURCADE, B. (2019), «L'ennemi iranien» dans BADIE, B. et VIDAL, D. (dir.), Fin du Leadership Américain ?, Paris, La Découverte, p. 198.
} 
États-Unis. Dans cette perspective, le 6 juin 2018, la Commission européenne lança la procédure d'activation de la loi dite «de blocage» afin de contourner les sanctions américaines ${ }^{1}$. Cet acte interdisait aux entreprises européennes de se conformer aux prescriptions américaines; dans le cas contraire, elles se verraient sanctionner par l'UE. Le mécanisme accordait donc une indemnisation aux entreprises touchées par la loi américaine mais il n'a pas abouti en raison de son caractère symbolique et n'a pas vraiment permis le contournement des sanctions américaines.

Dans la continuité de cette politique, l'UE a lancé une autre initiative pour reprendre le commerce avec l'Iran. En effet en 2019, elle a créé un nouveau mécanisme (Instex), fondé sur le principe du troc, et autorisant les entreprises européennes de commercer avec l'Iran en contournant les sanctions des États-Unis ${ }^{2}$. La mesure est entrée en phase opérationnelle en mars 2020 et a permis d'exporter du matériel médical vers l'Iran. Désormais, les entreprises européennes qui exporteront vers l'Iran seront créditées par l'Instex. Il faut préciser que la portée du mécanisme est limitée et ne concerne que quelques secteurs : le commerce des produits alimentaires, des médicaments et de fournitures humanitaires entre l'Iran et plusieurs pays européens. L'objectif principal de l'UE consiste à encourager l'Iran à ne pas quitter 1'Accord de 2015 et à respecter ses engagements à cet égard : " [q]uant aux Européens, ils tentent d'apaiser la situation à l'aide d'arguments diplomatiques et économiques $»^{3}$. Pour l'Iran ces mesures sont insuffisantes et n'apportent pas de solutions durables aux sanctions et il demande à l'UE de respecter ses engagements. Au regard de la situation actuelle, la solution de l'UE, par le mécanisme de l'Instex, ne concerne pour l'instant qu'une transaction dans le cadre d'une aide apportée par l'UE pour des motifs humanitaires et sanitaires dans le cadre de la pandémie du Covid 19, ce qui constitue un moyen de montrer au gouvernement iranien la différence de l'UE par rapport aux États-Unis. D'un point de vue politique, ce mécanisme prend une valeur symbolique et peut-être faudra-t-il attendre les prochaines élections aux États-Unis pour un changement éventuel de la politique de ceux-ci.

\section{Conclusion}

La présente étude avait pour objectif d'exposer la limite de puissance de l'UE dans le système international, et en particulier dans ses relations avec l'Iran. Après avoir analysé les différentes dimensions de cette puissance à travers plusieurs cas, nous nous sommes concentrés sur l'exemple du projet nucléaire iranien afin de mettre en exergue les divergences entre les États-Unis et l'UE sur le sujet, notamment depuis l'arrivée de Donald Trump à la MaisonBlanche. Pour aborder cette matière, nous avons mobilisé plusieurs concepts analytiques des Relations internationales : le concept de l'acteur, celui de la puissance douce (soft power) et celui de la puissance normative (normative power). Nous les avons utilisés afin d'examiner la capacité d'influence de l'UE dans le cadre de sa puissance normative à travers ses relations avec l'Iran et les États-Unis. Notre étude a montré que, depuis 1991, l’UE ambitionne un rôle important sur le plan des relations internationales. Dans cette perspective, elle participe à la médiation, à la gestion des crises, à l'instauration de la paix et à la démocratisation de plusieurs pays. Cependant, son rôle doit être mesuré en fonction du contexte, des conditions politiques et du facteur des rapports de force. Selon F. Petiteville, « l'UE demeure pourtant un acteur très 'conditionnel' de la résolution des conflits ${ }^{4}$. L'UE est une organisation régionale dont la puissance ne peut être comparée à celle d'un État. La puissance normative européenne trouve

\footnotetext{
1 Voir le site de référence sur les questionnes européennes (Tout l'Europe, comprendre l'Europe), (le 03/05/2019),(https://www.touteleurope.eu/actualite/accord-nucleaire-iranien-qu-est-ce-que-l-extraterritorialitedes-lois-americaines.html, Iran : que peut faire l'Europe face aux sanctions des Etats-Unis?.

${ }^{2}$ Ibid.

${ }^{3}$ HOURCADE, B. (2019), op. cit., p. 198.

${ }^{4}$ PETITEVILLE, F. (2016), op. cit., p. 130.
} 
un écho favorable dans les rapports extérieurs, en particulier avec les pays candidats à l'adhésion. Le cas des pays d'Europe orientale confirme cette perception devenue réalité dans le sens où c'est en acceptant les normes prévues par les critères de Copenhague qu'ils sont devenus membres de l'UE. Par ailleurs, on peut citer les pays des Balkans occidentaux, ou la Turquie, qui doivent réaliser des réformes structurelles afin de remplir les conditions d'adhésion. À travers ces cas, force est d'observer que l'UE peut être considérée comme un acteur de démocratisation dans une partie du monde. Mais dans certains cas, cette puissance normative rencontre des limites car les pays comme la Russie ou la Chine rejettent les normes européennes relatives à la question des droits humains. De surcroît, ils s'opposent à la conditionnalité des relations commerciales et économiques au respect de ces normes.

En ce qui concerne la limite de la puissance normative de l'UE à l'égard de l'Iran, nous avons examiné cette question à travers quatre périodes de leurs relations. 1) De 1992 à 1997 : l'UE a établi un dialogue critique soumis à cinq conditions. Durant cette période, nous avons observé une évolution en matières économique et commerciale. Mais la puissance normative s'est vue limitée par le refus iranien de reconnaître l'universalité des normes, en particulier celles relatives aux droits fondamentaux. 2) De 1998 à 2005 : cette période coïncide avec la présidence de Khatami qui se montra favorable à une partie des normes dans le cadre du dialogue global. On enregistra quelques progrès dans les rapports entre ces deux protagonistes. Cependant la réalisation des conditions du dialogue exigées par l'UE s'est heurté à la résistance des conservateurs du régime en Iran sous l'égide du guide de la Révolution. Par ailleurs, la reprise des activités nucléaires a empêché la conclusion d'un ACC entre l'UE et l'Iran. 3) 20052013 : avec l'arrivée au pouvoir d'Ahmadinejad en 2005, les relations entre l'UE et l'Iran sont entrées dans une phase de crise, et en dépit des négociations entre les représentants des deux parties, Téhéran a rejeté les conditions de l'UE qui mettait en doute la vocation pacifique du nucléaire iranien. Les conditions de l'UE concernant l'arrêt de l'enrichissement d'uranium ont été jugées inacceptables par le gouvernement iranien qui a mis en avant le principe de nonnégociabilité sur ce sujet. C'est pourquoi l'UE a changé sa stratégie à l'égard de l'Iran à partir de 2010 par l'application d'une diplomatie coercitive. Cette période coïncide aussi avec la montée en puissance de l'Iran à l'échelle régionale et cette ascension a joué un rôle dans le processus des négociations. Par conséquent, la crise nucléaire a complètement influencé le dialogue irano-européen et a marginalisé la question des droits humains. 4) 2013-2020 : en 2013 avec l'arrivée au pouvoir d'Hassan Rohani, l'UE a mobilisé toute sa capacité diplomatique afin de jouer un rôle de médiateur dans les négociations multilatérales entre l'Iran et le groupe P5+1. Durant cette période, les États-Unis et l'UE ont partagé la même approche pour reconnaître le droit de l'Iran au nucléaire civil. Ce changement politique a joué un rôle crucial dans la conclusion de l'Accord de 2015 qui fut un succès pour la " politique étrangère commune européenne ». Par ailleurs, les activités diplomatiques des représentants de l'UE auprès de la Russie et de la Chine ont permis de rapprocher les positions de ces acteurs avec celles des ÉtatsUnis. Cette évolution s'accorda à l'existence d'un intérêt commun auprès des acteurs concernés. Ce qui a permis à l'UE d'exploiter sa capacité d'influence politique et diplomatique dans le processus des négociations. Certes, les États-Unis ont pris le leadership dans ces négociations mais l'UE a joué un rôle complémentaire. Selon Clément Therme : «à partir de l'année 2007, nous assistons à l'alignement progressif des capitales européennes sur la ligne américaine. De plus, l'élection de Barack Obama se traduit par un revirement diplomatique de Washington qui surprend les Européens et les pousse vers une responsabilité formelle de la négociation mais sous la tutelle américaine $» 1$.

Par conséquent, cet Accord a montré que, lorsque la politique européenne présente une certaine cohérence, l'UE peut devenir un acteur des résolutions des crises et des conflits. Cet

${ }^{1}$ THERME, C. (2020), « Le nucléaire iranien vu de France », Confluences méditerranée, n 113, l’Harmattan, p, 106. 
Accord a permis, quelques mois plus tard, la levée progressive de l'embargo et la normalisation des relations avec l'Iran. Mais l'élection de Donald Trump a complètement changé la politique des États-Unis au sujet de l'Accord. En 2018, les États-Unis s'en sont retirés et ont imposé leur loi extraterritoriale aux entreprises étrangères en interdisant le commerce avec l'Iran. Malgré sa protestation, l'UE n'a pas pu empêcher que cette interdiction s'impose aux entreprises européennes. De ce fait, cette confrontation, diplomatique et commerciale, a révélé la limite de la puissance normative de l'UE et sa dépendance vis-à-vis de Washington.

\section{Bibliographie}

Agence France Presse, le 26 octobre 2010.

ARON, R. (1962), Paix et guerre entre les nations, Paris, Calmann-Lévy.

BADIE, B. (2019), L'Hégémonie contestée. Les nouvelles formes de domination internationale, Paris, Odile Jacob.

BATTISTELLA, D. (2011), Paix et Guerres au XXI siècle, Paris, Sciences Humaines Editions.

BATTISTELLA, D. (2012), Théories des relations internationales, Paris, Sciences Po.

BATTISTEllA, D., SMOUT, M-C., VENNESSON, P., PETITEVILlE, F. (2006), Dictionnaire des relations internationales, Paris, Dalloz.

BAYRAMZADEH, K. (2004), Les enjeux principaux des relations entre l'Iran et l'Europe de 1979 à 2003, Paris, l'Harmattan.

BAYRAMZADEH, K. (2013), «La lutte pour l'hégémonie régionale dans les relations internationales : le cas du conflit syrien », Cahiers de Sciences politiques de l'ULG, Cahier $\mathrm{n}^{\circ} 27$, disponible à l'adresse suivante : http://popups.ulg.ac.be/csp/ (consultée le 13 juillet 2020).

BLACHER, P. (2006), Droit des relations internationales, Paris, Litec.

BONIFACE, P. (2019), Comprendre le monde, Paris, Armand Collin.

CABASTAN, J-P. (2015), La politique internationale de la Chine, Paris, Sciences Po.

CIZEL, A. (2020), «L'obsession iranienne: les Etats-Unis au défi des (dès)équilibres régionaux », in Clément Therme, L'Iran et ses rivaux, Passés composés.

Communication de la Commission au Conseil et au Parlement européen, Relations EURépublique Islamique de l'Iran, le 7 février 2001.

CORDESMAN, A. (1997), Iran: Dilemmas of dual containment, New York, Westview Press.

DEVIN, G. (2013), Sociologie des relations internationales, Paris, La Découverte. 
DJALILI, M-R. (2013-2014), «Politique étrangère de la République islamique : le rôle du président », Confluences méditerranée, $\mathrm{n}^{\circ} 88$, Paris, 1'Harmattan.

DUCHENE, F, (1972), «Europe's role in world peace », in Richard Mayen (ed.), Europe tomorrow. Sixteen Europeans look ahead, London,

DUFAYS, N. (2012), «Les relations de l'Union européenne avec la République islamique de l'Iran : enjeux, perspectives et perceptions », dans MAKINSKY, M. (dir.), L'Iran et les grands acteurs régionaux et globaux, Paris, L'Harmattan.

EIFFLING, V. (2013), «Les États-Unis face à la question nucléaire iranienne : un nœud gordien perpétuel ? », dans NAHAVANDI, F. (dir.), L'Iran dans le monde, Paris, l'Harmattan.

FIEDLER, R. (2018), «Iran and the European Union after the Nuclear Deal », CES Working Papers, Alexandru Ioan Cuza University of Iasi, Centre for European Studies, Iasi, vol. 10, Issue 3.

GAHLER, M. (2018), Le rapport du parlement européen sur les relations entre l'UE et la République islamique, Bruxelles, le 26 novembre 2001.

HOURCAD, B. (2019), «L'ennemi iranien» dans BADIE, B. et VIDAL, D. (dir.), Fin du Leadership Américain?, Paris, La Découverte.

JUPILLE, J. and CAPORASO, J. (1998), « States, Agency and Rules: The European Union in Global Environmental Politics » in RHODES, C. (ed.), The European Union in the World Community, Boulder, Colorado, Lynne Rienner.

KAGAN, R. (2004), La puissance et la faiblesse : Les États-Unis et l'Europe dans le nouvel ordre mondial, Paris, éd. Hachette, coll. Pluriel.

KANDAL, M. (2018), «États-Unis-Arabie Saoudite, une alliance ambiguë », Questions internationales, Paris, La documentation française, $\mathrm{n}^{\circ} 89$.

KEMP, G. (1998), « The United States, Iran and Irak: Containment or Engagement? », US Policy and Iran, Paris, Edition Ifri.

La déclaration de sir Leon Brittan, le 30 septembre 1997, Bruxelles, Source d'Europe, 31494.

LAÏDI, Z. (2009), «L'Europe, puissance normative internationale », dans DEHOUSSE, R., Politiques européennes, Paris, Presse de Sciences Po.

LAROCHE, J. (2011), La brutalisation du monde : du retrait des États à la décivilisation, Québec, Liber.

LEFEBVERE, M. (2011), La politique étrangère européenne, Paris, PUF.

LEMAITRE, P. (1997), « L'Europe gèle le « dialogue critique » avec l'Iran mais ne prend pas de sanctions économiques », Le Monde, le 2 mai 1997, https://www.lemonde.fr/archives/article/1997/05/02/l-europe-gele-le-dialogue-critique-avec-liran-mais-ne-prend-pas-de-sanctions-economiques_3540827_1819218.html (consulté le 15 janvier 2021). 
LESNES, C. (2013), «La politique étrangère de Barack Obama : la tentation du repli ? », dans BADIE, B. et VIDAL, D. (dir.), Puissances d'hier et de demain, La Découverte.

LIKA, L. (2016), «La pénétration turque dans les Balkans occidentaux : quels défis pour le projet d'élargissement de l'UE ?», dans SANTANDER, S. (dir.), Concurrences régionales dans un monde multipolaire émergent, Bruxelles, P.I.E. Peter Lang.

LOISEAU, H. (2009), «Les approches contemporaines des relations internationales », dans PAQUIN, S. et DESCHENES, D. (dir.), Introduction aux relations internationales, Canada, Chenelière Education.

MAKINSKY, M. (2016), «Iran-Arabie Saoudite : quel dialogue après l'accord nucléaire ?», Stratégique Orients, ${ }^{\circ} 3$, l'Harmattan.

MANNERS, I. (2002), « Normative Power Europe: A contradiction in Terms? », Journal of Common Market Studies, vol. 40, $\mathrm{n}^{\circ} 2$.

MEARSHEIMER, J. (2014), The Tragedy of Great Power Politics, USA, University of Chicago.

MEARSHEIMER, J. (2019), «Bound to Fail, The Rise and Fall of the Liberal International Order », Security, vol. 43, n 4.

Mehr News (site web iranien) : www.mehrnews.com/fa/ (26.11.2008).

MERLE, M. (1988), Sociologie des relations internationales, Paris, Dalloz.

MICHEL, L. (2009), «La refonte de la stratégie de défense américaine », Revue Internationale et Stratégique, $\mathrm{n}^{\circ} 76$.

MIKHAÎL, B. (2010), «Une diplomatie du pragmatisme aux effets limités au MoyenOrient ? », Revue Internationale et Stratégique, $\mathrm{n}^{\circ} 77$.

MORGENTHAU, H. (1948), Politics Among Nations. The struggle for Power and Peace, New York, Knopf.

NICOULLAUD, F. (2016), «La fin d'un bras de fer avec la communauté internationale ? Retour sur douze années de négociations nucléaires », Iran le retour, Questions internationales, $\mathrm{n}^{\circ} 7$, La documentation française.

NYE, J. (1990), Bound to lead. The Changing Nature of American Power, New York, Basic Books Publishers.

PAQUIN, S. (2009), «L'économie politique internationale et la mondialisation », dans PAQUIN, S. et DESCHÈNES, D. (dir.), Introduction aux relations internationales. Théories, pratiques et enjeux, Montréal, Chenelière.

PETITEVILLE, F. (2002), «L'Union européenne, un acteur international 'global' », Revue internationale et stratégique, $\mathrm{n}^{\circ} 43$. 
PETITEVILLE, F. (2006), La politique internationale de l'Union européenne, Sciences Po.

PETITEVILLE, F. (2016), «L'Union européenne, acteur conditionnel de la résolution des conflits », dans BAZIN, A. et TENENBAUM, C. (dir.), L'Union européenne et la paix, Paris, Presses de Siences Po.

ROSENEAU, J. (1990), Turbulence in World Politics. A Theory of Change and Continuity, Princeton, Princeton University Press.

ROUSSELET, L. (2017), Négocier l'Atome : Les États-Unis et les négociations de l'accord sur le nucléaire iranien, Paris, l'Harmattan.

SANTANDER, S. (2016) (dir.), Concurrences régionales dans un monde multipolaire émergent, Bruxelles, P.I.E. Peter Lang.

SANTANDER, S. VLASSIS, A. (2020), «EU in Global Affairs: Constrained Ambition in an Unpredictable World? », European Foreign Affairs Review, vol. 25, $\mathrm{n}^{\circ} 1$.

SANTANDER, S., VLASSIS, A. (2018), «L'UE, une puissance commerciale bousculée », Diplomatie, $\mathrm{n}^{\circ} 47$.

SCANDELLA, J. (2005), «L'Union européenne et l'Iran : entre institutionnalisation et suspension des relations », Les Cahiers de l'Orient, $\mathrm{n}^{\circ} 79$.

THERME, C. et KHAZANEH, R. (2012), «Le développement du programme nucléaire iranien : entre aspects techniques et questions politiques », Maghreb-Machrek, n 212.

THERME, C. (2020), « Le nucléaire iranien vu de France », Confluences méditerranée, $\mathrm{n}^{\circ} 113$, l'Harmattan.

VAN ENGELAND-NOURAI, A. (2005), «Le rôle des droits de l'homme dans les relations entre l'Union européenne et l'Iran », Les Cahiers de l'Orient, $\mathrm{n}^{\circ} 79$.

VIAUD, A. (2017), L'Union européenne face à la crise nucléaire iranienne, Belgique, Presses universitaires de Louvain.

WANG, Y. (2009), «The Identity Dilemmas of EU Normative Power: Observations from Chinese Traditional Culture », in GERRITS André (ed.), Normative Power Europe in a Changing World: A Discussion, The Hague, Netherlands, Clingedael European Papers $\mathrm{n}^{\circ} 5$.

ZARIFIAN, J. (2015), « Le dossier nucléaire iranien et la question du rapprochement ÉtatsUnis-Iran », Politique américaine, $\mathrm{n}^{\circ} 26$. 